\title{
Applying an innovative biodegradable self-assembly nanomicelles to deliver a-mangostin for improving anti-melanoma activity
}

Shuping Yang ${ }^{1}$, Xiang Gao ${ }^{1}$, Yihong $\mathrm{He}^{1}$, Yuzhu Hu${ }^{1}$, Bocheng $\mathrm{Xu}^{1}$, Zhiqiang Cheng $\mathbb{\circledR}^{2}$, Mingli Xiang ${ }^{1}$ and Yongmei Xie (1) ${ }^{1}$

\begin{abstract}
a-Mangostin (aM), a traditional natural product with promising application of treating a series of diseases, was limited use in clinical due to its hydrophobicity. Herein, MPEG-PCL nanomicelles were used to embed the aM for resolving hydrophobicity and improving the anti-melanoma effect of the aM. The anti-melanoma activity and potential mechanisms of biodegradable aM/MPEG-PCL nanomicelles were investigated. The aM/MPEG-PCL nanomicelles possessed a stronger effect on anti-melanoma compared to the free aM both in vitro and in vivo with a low cytotoxicity in non-tumor cell lines. In the research of mechanisms, the aM/MPEG-PCL nanomicelles inhibited the proliferation of melanoma cell, induced apoptosis via both apoptosis pathways of intrinsic and exogenous in vitro, as well as suppressed tumor growth and restrained angiogenesis in vivo, which implied that the aM/MPEG-PCL nanomicelles have potential application as a novel chemotherapeutic agent in melanoma therapy.
\end{abstract}

\section{Introduction}

Nanotechnology, an international gold rush, is a revolutionizing and rapidly evolving technology inspiring many areas in this word ${ }^{1,2}$. The progress and exploitation of nanotechnology in medicine have conquered tremendous breakthrough in human health career $^{3,4}$. Definitely, the effective nanotechnology is of great significance in the improvement of nanomedicine which has received extensive attention in application of gene therapy, personalized treatments, and precision medicine for oncotherapy $^{5-7}$, notably the development of new drug delivery

\footnotetext{
Correspondence: Xiang Gao (xianggao@scu.edu.cn) or

Mingli Xiang (xiangmingli@scu.edu.cn) or Yongmei Xie (xieym@scu.edu.cn)

'Department of Neurosurgery and Institute of Neurosurgery, State Key Lab of Biotherapy and Cancer Center, West China Hospital, Sichuan University and Collaborative Innovation Center for Biotherapy, Chengdu 610041, PR China ${ }^{2}$ Department of Pharmacology and Molecular Sciences, Johns Hopkins

University School of Medicine, Baltimore, MD 21205, USA

These authors contributed equally: Shuping Yang, Xiang Gao

Edited by S. Tait
}

systems in cancer treatment. At present, several drug delivery systems have been reported, including solid dispersion, multistage drug delivery systems (Ms-DDS), phospholipid complex and so forth ${ }^{8-10}$. However, many defects exist in terms of stability, effectiveness, and preparation. Nanotechnology is a new technique for the application of nanocarriers to encapsulate hydrophobic drugs to improve the water solubility. Nanometer medicine carriers have been diversely developed, such as liposomes, lipids, nanomicelles, silica, and iron oxide. Among them, the applications of biodegradable nanomicelles have received comprehensive attention ${ }^{11-13}$. It is a novel technique for the application of nanocarriers to encapsulate hydrophobic drugs to improve its water solubility and strengthen efficacy ${ }^{12,13}$.

Notoriously, the most devastating form of skin cancer is melanoma with a highly metastatic potential ${ }^{14,15}$. The rate of annual increment is obvious in the last 30 years in the USA, meanwhile, melanoma possesses rapidly increasing incidence

\section{(c) The Author(s) 2019}

(c) (i) Open Access This article is licensed under a Creative Commons Attribution 4.0 International License, which permits use, sharing, adaptation, distribution and reproduction cc) in any medium or format, as long as you give appropriate credit to the original author(s) and the source, provide a link to the Creative Commons license, and indicate if changes were made. The images or other third party material in this article are included in the article's Creative Commons license, unless indicated otherwise in a credit line to the material. If material is not included in the article's Creative Commons license and your intended use is not permitted by statutory regulation or exceeds the permitted use, you will need to obtain permission directly from the copyright holder. To view a copy of this license, visit http://creativecommons.org/licenses/by/4.0/. 
a<smiles>COc1c(O)cc2oc3cc(O)c(CCC(C)=O)c(O)c3c(=O)c2c1CC=C(C)C</smiles>

c

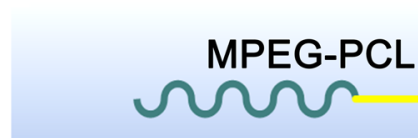

$\mathrm{aM}$

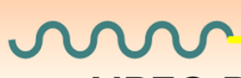

MPEG-PCL b

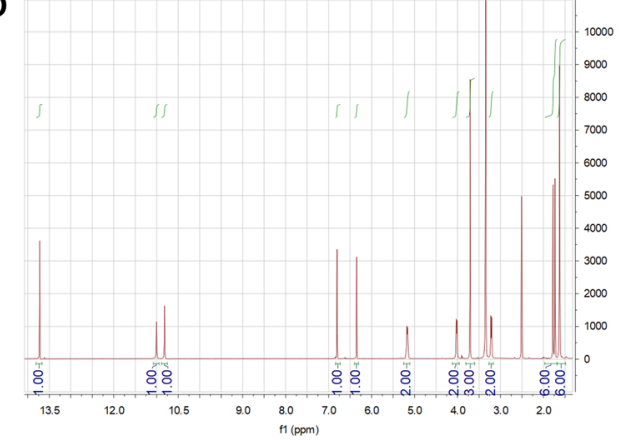

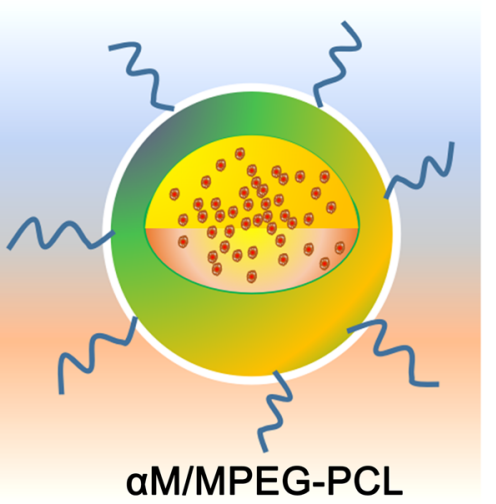

Fig. 1 The chemical information of the aM and preparation of the aM/MPEG-PCL micelles. a The chemical structure of the aM. $\mathbf{b}^{1} H N M R$ of the $\mathrm{aM}$. c Preparation scheme of the aM/MPEG-PCL nanomicelles. The free $\mathrm{aM}$ and the MPEG-PCL self-assembled into the aM/MPEG-PCL nanomicelles

rate in whole-world ${ }^{16}$. In 2017, there were 87,110 new cases of melanoma and 9730 deaths associated with the melanoma in the USA ${ }^{17}$. The melanomas possess the highest mutational load in all human cancers, because there are hundreds to thousands of mutations per tumor ${ }^{18}$. However, it is difficult to find a satisfactory therapeutic regimen to treating the melanoma. The major allure of nanomedicine is the possibility of faster and more advanced routes to the settlement of the most pressing and the hardest medical problems. Therefore, one of the more promising ways to treat melanoma is the application of nanomedicine.

As an exotic fruit, mangosteen was found in South Africa and its peel had used for centuries as a traditional medicine to cure inflammation, ulcer, skin infection, wound healing, and other diseases ${ }^{19}$. $\alpha$-Mangostin $(\alpha M)$ (Fig. 1a), the major xanthone derivative, is the most important secondary metabolite extracted from the pericarp of mangosteen ${ }^{19-21}$. Meanwhile, the $\alpha M$ was reported with several significant biological activities to benefit health promotion, such as anti-tumor, anti-inflammatory, anti-virus, anti-bacterial, anti-fungal, antioxidant, heart protection, and so on ${ }^{22-25}$. However, the $\alpha M$ is a strong oleophilic compound with poor aqueous solubility ${ }^{25}$, which limits its clinical applications.

In this study, we selected biodegradable nanomicelles of monomethoxy poly (ethylene glycol)-polycaprolactones
(MPEG-PCLs) to wrap the $\alpha M$ to improve its water solubility. More significantly, we investigated the efficiency of the $\alpha M / M P E G-P C L$ nanomicelles on the therapy of the melanoma in vitro and in vivo and explored the potential anti-tumor mechanisms.

\section{Results}

\section{Preparation of the aM/MPEG-PCL nanomicelles}

The $\alpha M$ nanomicelles were prepared by a selfassembly method to improve the water solubility. Briefly, the $\alpha M$ and the MPEG-PCL were co-dissolved in anhydrous methanol. After that, the solution was evaporated in rotary evaporator to promote the $\alpha \mathrm{M}$ distributed into the MPEG-PCL copolymer. The mixture was dissolved in normal saline solution, which allowed the $\alpha M$ self-assembled into the MPEG-PCL nanomicelles. As shown schematically in Fig. 1c, the $\alpha M /$ MPEG-PCL possessed a core-shell structure with PCL (the hydrophobic segment) as a core to collect $\alpha M$, and MPEG (the hydrophilic segment) as a shell in aqueous solution.

\section{Molecular modeling study of interaction between the aM and MPEG-PCL copolymer}

As demonstrated in Fig. 2, both the $\alpha \mathrm{M}$ and the copolymer MPEG-PCL were trying to adjust their 


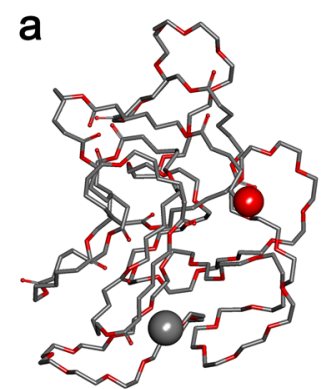

C

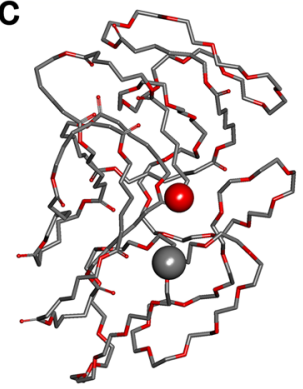

e

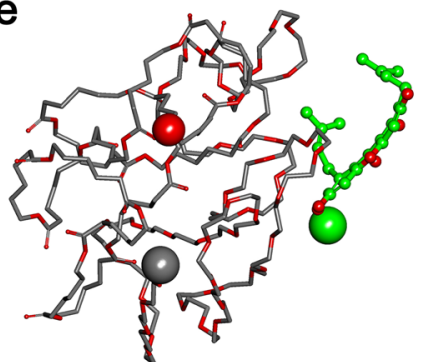

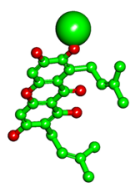

d

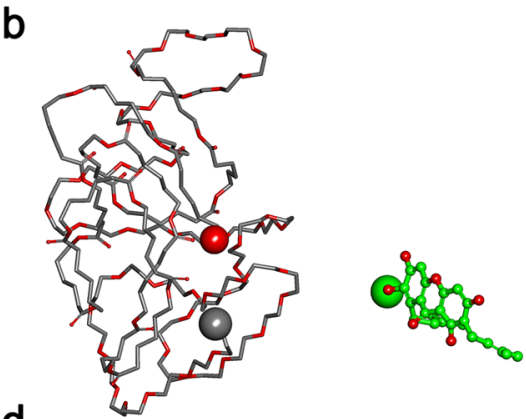

f
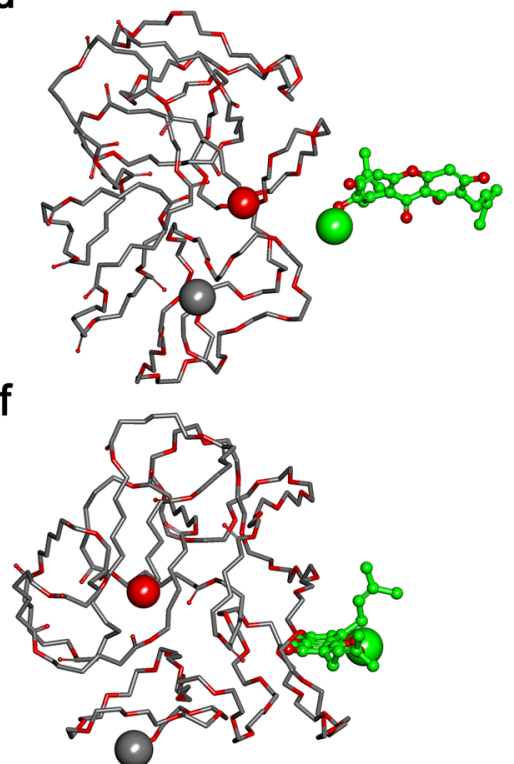

Fig. 2 Interaction modes between polymer and compound revealed by Langevin dynamics simulation in an aqueous environment. a The initial conformation of the polymer MPEG-PCL complexed with the $\mathrm{aM}$; Conformations $\mathbf{b}, \mathbf{c}, \mathbf{d}, \mathbf{e}$, and $\mathbf{f}$ are corresponding to snapshots of the complex collected at 9.985 ps, 15.995 ps, 20.020 ps, 29.945 ps, and 100 ps, respectively. The polymer MPEG-PCL is represented with thick stick, whereas the $\mathrm{aM}$ is depicted with ball and stick style and its carbon atoms are colored with green. Two terminal heavy atoms in the polymer MPEG-PCL is highlighted using a "ball" style, and the head heavy atom in the aM are highlighted using CPK style

conformations and shorting the distance between them so as to gain a favorable interaction mode.

An interesting result was observed by comparing the simulation of the complex in water and the simulation in tumor. It was shown in Fig. 3 that in these two kinds of different environment, the interaction modes between the $\alpha M$ and the MPEG-PCL were different. In the aqueous environment, copolymer in the $\alpha \mathrm{M} / \mathrm{MPEG}-\mathrm{PCL}$ complex tended to be a globe shape and the binding site was more suitable for interaction with the $\alpha \mathrm{M}$. While in tumor environment, the copolymer seemed not to keep a globe shape and the mouth of the binding pocket was more open that must be easier for the $\alpha \mathrm{M}$ to escape from a pocket. In another word, the interaction between the $\alpha \mathrm{M}$ and the MPEG-PCL in tumor was weaker than that in water.

\section{Characterization of the aM/MPEG-PCL nanomicelles}

The average particle size, polydispersity index, zeta potential, and appearance of $\alpha$ M/MPEG-PCL nanomicelles were further determined and presented. In Fig. 4a, the size distribution spectrum of the freshly prepared $\alpha M / M P E G-P C L$ nanomicelles showed a very narrow particle size distribution (mean particle size $=30 \mathrm{~nm}$ ) as a monodisperse system. According to Fig. $4 \mathrm{~b}$, the $\alpha \mathrm{M} /$ MPEG-PCL nanomicelles had a zeta potential of $-2.1 \mathrm{mv}$. Furthermore, transmission electron microscopy (TEM) was applied to study the morphology of the $\alpha \mathrm{M} / \mathrm{MPEG}$ PCL, which revealed the $\alpha M / M P E G-P C L$ nanomicelles were spherical with an average diameter of $23 \mathrm{~nm}$ in drying phase (Fig. 4c). High-performance liquid chromatography (HPLC) assay illustrated that $\alpha$ M/MPEG-PCL nanomicelles had the drug loading (DL) of $99.1 \%$ and encapsulation efficiency (EE) of $10 \%$.

\section{In vitro release analysis}

The in vitro release results indicated the $\alpha M$ was released more slowly from the $\alpha \mathrm{M} / \mathrm{MPEG}-\mathrm{PCL}$ nanomicelles compared to the free $\alpha \mathrm{M}$ via dialysis under sink 

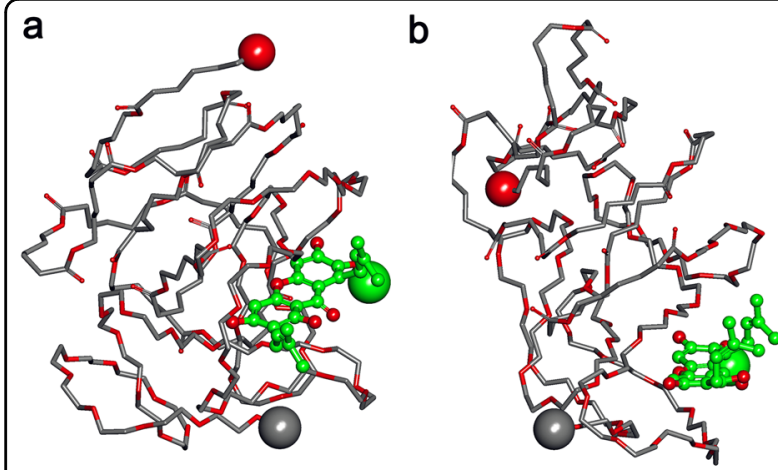

C
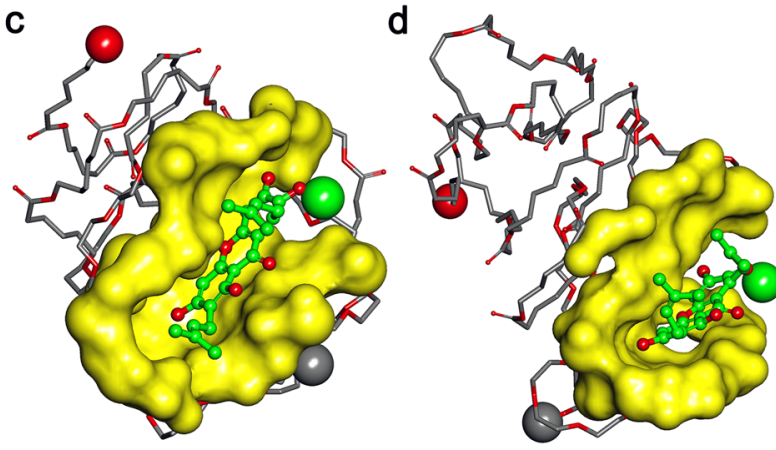

Fig. 3 Interaction modes between polymer and compound revealed by Langevin dynamics simulation in water and tumor environment. The demonstration style is the same as in Fig. 2. a The conformation of the polymer MPEG-PCL complexed with the aM after being simulated in water environment for 200 ps; b The conformation of the polymer MPEG-PCL complexed with the aM after being simulated in water environment for $100 \mathrm{ps}$ and then in tumor environment for $100 \mathrm{ps}$; $\mathbf{c}$ and $\mathbf{d}$ are corresponding to $\mathbf{a}$ and $\mathbf{b}$, but the binding site is highlighted with solid surface and colored light yellow

condition with a $0.5 \%$ Tween $80(\mathrm{w} / \mathrm{w})$ solution in phosphate-buffered saline (PBS, $\mathrm{pH}=7.4$ ). As shown in Fig. $4 d$, the $\alpha M / M P E G-P C L$ nanomicelles indicated a much slower cumulative release rate compared to the free $\alpha \mathrm{M}$. During the first $24 \mathrm{~h}$, only $25.3 \pm 2.48 \%$ of the $\alpha \mathrm{M}$ was released into the media in the nanomicelles group, whereas $53.8 \pm 0.85 \%$ of the $\alpha \mathrm{M}$ was released in the freedrug group. At the $72-\mathrm{h}$ point, $91.5 \% \pm 2.55$ and $43 \% \pm$ $0.88 \%$ of the $\alpha \mathrm{M}$ were released from the free-drug group and the $\alpha M / M P E G-P C L$ nanomicelles, respectively. Therefore, the $\alpha M / M P E G-P C L$ nanomicelles demonstrated a sustained drug-release profile compared with the free drug.

\section{In vivo pharmacokinetics analysis}

Bioavailability improvement by increasing hydrophilicity is the major objective of the encapsulation of the $\alpha M$ into the MPEG-PCL nanomicelles. To prove this hypothesis, we analyzed the mean plasma concentrations of the $\alpha \mathrm{M}$ after the tail intravenous injection of the free drug and the nanomicelles drug. As presented in
Fig. 4e, the free $\alpha M$ group was fleetly cleared with a plasma level of the $\alpha \mathrm{M}$ of $3.26 \pm 0.44 \mu \mathrm{g} / \mathrm{mL}$ compared with $145.34 \pm 35.56 \mu \mathrm{g} / \mathrm{mL}$ in the group of the $\alpha \mathrm{M} /$ MPEG-PCL nanomicelles after injection over $5 \mathrm{~min}$. Apparently, the tremendous difference existed after the $2 \mathrm{~h}$ incubation, which proved that the MPEG-PCL nanomicelles significantly reduced the $\alpha M$ elimination.

\section{Effects on the cell proliferation of melanoma cells and non-} tumor cell lines

The anti-cancer activities of the $\alpha M$ and the $\alpha M /$ MPEG-PCL nanomicelles were studied by 3-(4,5-dimethylthiazol-2-yl)-2,5-d/iphenyltetrazolium bromide (MTT) assay. Briefly, A375 and B16 melanoma cells were treated with gradient concentrations of the $\alpha \mathrm{M}$ and $\alpha \mathrm{M} /$ MPEG-PCL nanomicelles from 0 to $100 \mu \mathrm{g} / \mathrm{mL}$ for $24 \mathrm{~h}$, $48 \mathrm{~h}$ and $72 \mathrm{~h}$, respectively. As shown in Fig. 5a, in the lower concentrations, both the $\alpha M$ and $\alpha M / M P E G-P C L$ nanomicelles exhibited no significant inhibition on cell viability of A375 and B16 cells. Subsequently, the suppression effects of the $\alpha M$ and the $\alpha M / M P E G-P C L$ nanomicelles had no obvious dose dependent in the higher concentrations. However, the $\alpha \mathrm{M} / \mathrm{MPEG}-\mathrm{PCL}$ nanomicelles possessed stronger inhibitory effect compared to the $\alpha \mathrm{M}$. In the effectual concentrations, the cell viability of A375 and B16 cells treated with the $\alpha M /$ MPEG-PCL nanomicelles were weaker than the one treated with the $\alpha M$. These results indicated that the embedding of MPEG-PCL improved the cytotoxicity of the $\alpha M$ to the melanoma cells. To further validate and compare the growth inhibitory effect of the $\alpha \mathrm{M}$ and the $\alpha \mathrm{M} / \mathrm{MPEG}-\mathrm{PCL}$ nanomicelles. A375 and B16 cells, clonogenic assay was performed. As shown in Fig. 5b, with the increasing concentrations of the $\alpha M$ and the $\alpha M /$ MPEG-PCL nanomicelles, the size of the colony formation of the melanoma cells obviously reduced. Moreover, no colony formation was observed in A375 cells plate treated with $12.5 \mu \mathrm{g} / \mathrm{mL}$, as well as B16 cells plate treated with $6.25 \mu \mathrm{g} / \mathrm{mL}$ in the $\alpha \mathrm{M} / \mathrm{MPEG}-\mathrm{PCL}$ nanomicelles group, nevertheless, several colony formations under the same condition were formed in the $\alpha \mathrm{M}$ parallel experiments. These results further demonstrated the $\alpha M /$ MPEG-PCL nanomicelles had a more intense restrain effect on the level of colony formation in melanoma cells compared to the $\alpha \mathrm{M}$.

Meanwhile, non-tumor cell lines, including LO2, Vero, and HEK293T cells, were treated by the same condition. The MTT results demonstrated the $\alpha M / M P E G-P C L$ nanomicelles $(<12.5 \mu \mathrm{g} / \mathrm{mL})$ exhibited low a cytotoxicity for non-tumor cell lines in Fig. 5c. Indeed, the cell viabilities were more than $50 \%$ and $20 \%$, respectively, when it was treated by $12.5 \mu \mathrm{g} / \mathrm{mL}$ and $25 \mu \mathrm{g} / \mathrm{mL}$ the $\alpha \mathrm{M} /$ MPEG-PCL nanomicelles. 


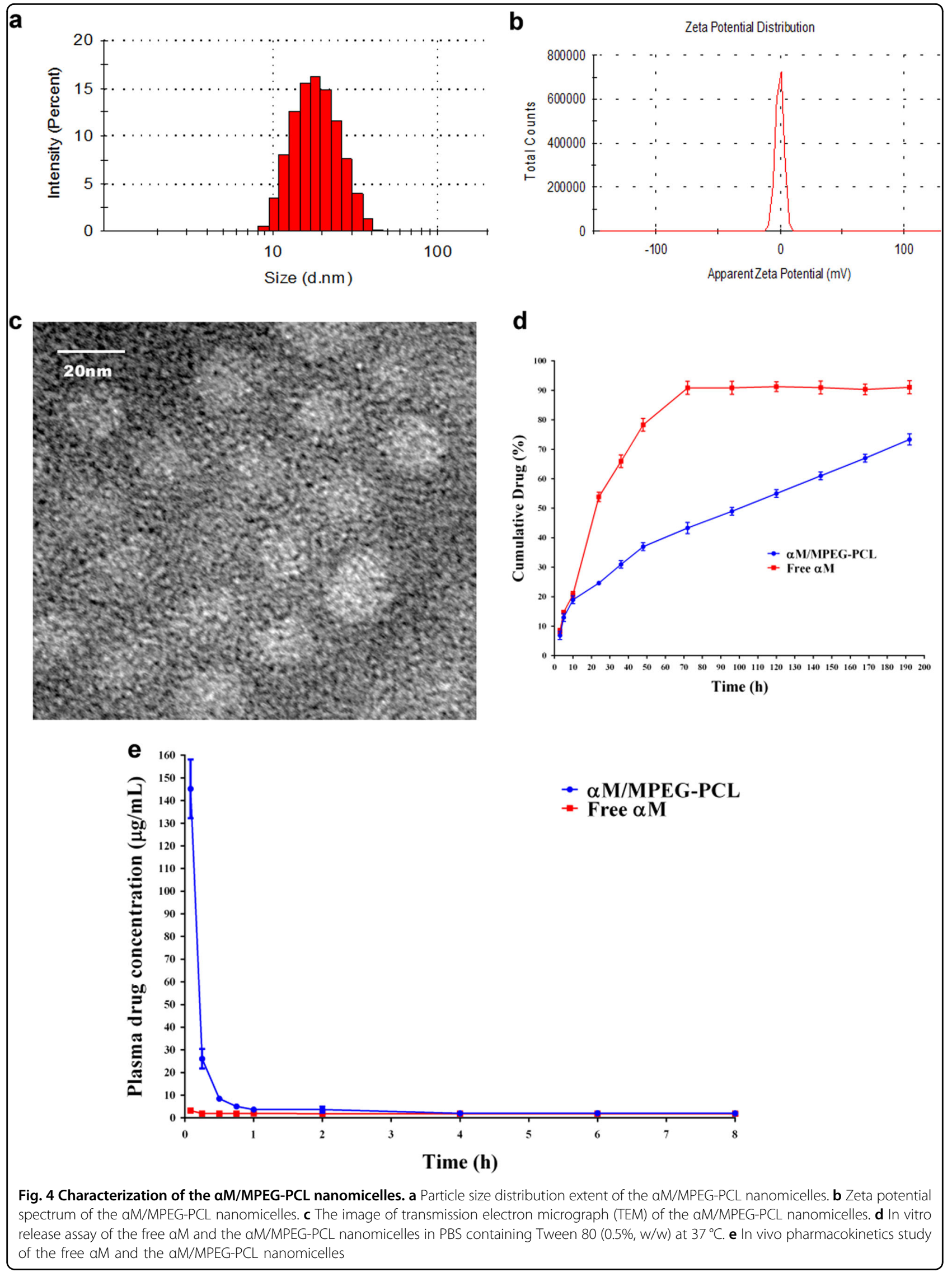




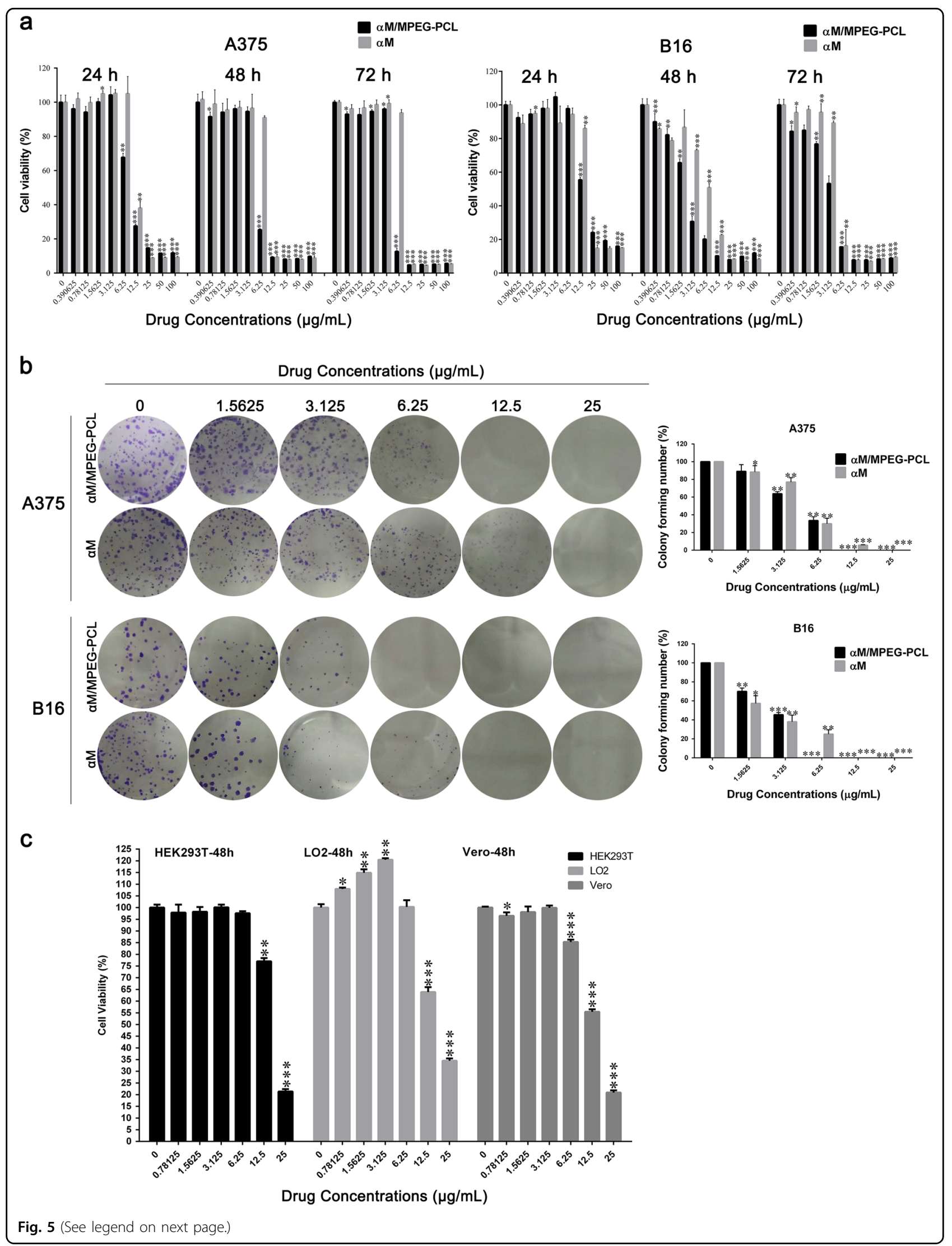


(see figure on previous page)

Fig. 5 Effects of the free $\mathbf{a M}$ and the aM/MPEG-PCL nanomicelles on the cell proliferation. Cell viability was measured with MTT assay. a Cell viability of melanoma cells treated with various concentrations of the free aM and the aM/MPEG-PCL nanomicelles for $24 \mathrm{~h}, 48 \mathrm{~h}$, and $72 \mathrm{~h}$. Values represent mean $\pm \mathrm{SD}\left(n=3\right.$, in triplicate, ${ }^{*} P<0.05$; ${ }^{*} P<0.01 ;{ }^{* *} P<0.001$ compared to control). $\mathbf{b}$ The effects of the free aM and the aM/MPEG-PCL nanomicelles on colony formation in melanoma cells and the statistic assay of colony formation experiments. Data are expressed as mean \pm SD (from three independent experiments), ${ }^{*} P<0.05 ;{ }^{* *} P<0.01$; ${ }^{* *} P<0.001$ compared to vehicle $(0 \mu \mathrm{M}$ group). c Non-tumor cell lines (LO2, Vero, and HEK293T) were incubated with a series of concentrations of the aM/MPEG-PCL nanomicelles for $48 \mathrm{~h}$. Values represent mean \pm SD $(n=3$, in triplicate, ${ }^{*} P<0.05 ;{ }^{* *} P<0.01 ;{ }^{* *} P<0.001$ compared to control)

\section{The mechanisms of the anti-melanoma effect of the aM/} MPEG-PCL nanomicelles in vitro

To study the apoptosis efficiency induced by the $\alpha \mathrm{M}$ and the $\alpha \mathrm{M} / \mathrm{MPEG}-\mathrm{PCL}$ nanomicelles, A375 cells were studied by flow cytometry (FCM) using Annexin V-FITC/ PI staining. According to Fig. 6a, the A375 cells apoptosis rates were calculated to $6.58 \%$ (free $\alpha M$ group) and $5.99 \%$ $(\alpha M / M P E G-P C L$ nanomicelles group) in the negative control. However, in the $\alpha M$ and the $\alpha M / M P E G-P C L$ nanomicelles groups, the cell apoptosis rates were enhanced with the increasing of concentrations of the $\alpha \mathrm{M}$ and the $\alpha M / M P E G-P C L$ nanomicelles. Moreover, the cell apoptosis rates were calculated to $68.9 \%$ and $33.6 \%$ in the $\alpha M / M P E G-P C L$ nanomicelles and the $\alpha M$ group, respectively, after $24 \mathrm{~h}$ treatment by a concentration of $12.5 \mu \mathrm{g} / \mathrm{mL}$. Obviously, the $\alpha \mathrm{M} / \mathrm{MPEG}-\mathrm{PCL}$ nanomicelles possessed more pronounced effect on the A375 cell apoptosis induction compared to the $\alpha \mathrm{M}$.

To explore the reason that the $\alpha M / M P E G-P C L$ micelles could enhance apoptosis induction, we compared the cellular uptake ability of the $\alpha M / M P E G-P C L$ nanomicelles and the $\alpha M$ in A375 cells. The HPLC assay results in Fig. $6 b$ clearly demonstrated the $\alpha M / M P E G-P C L$ nanomicelles had a better cellular uptake behavior than the $\alpha M$.

Since, the loss of mitochondrial potential is a significant event in the intrinsic apoptotic pathway, the mitochondrial membrane potential was performed for an investigation on the apoptotic pathway activated by the $\alpha \mathrm{M} /$ MPEG-PCL nanomicelles. The Fig. $6 \mathrm{c}$ showed that the Rh123 accumulation in A375 and B16 cells treated with the $\alpha M / M P E G-P C L$ nanomicelles were cut down compared with the control. Indeed, the specific differences were presented as a concentration-dependent manner.

Additionally, several proteins-related mitochondrial apoptosis pathway were analyzed. After incubating with gradient concentrations of the $\alpha M / M P E G-P C L$ nanomicelles, A375 cells were lysed. The expression levels of Bax and Bcl-2 tested by western blot were summarized in Fig. 6d. The level of Bcl-2 was markedly reduced in a dose-dependent manner, while the level of Bax increased. Additionally, these results implied that the $\alpha \mathrm{M} / \mathrm{MPEG}$ PCL nanomicelles-induced apoptosis in melanoma cells by the mitochondrial apoptosis pathway.
Moreover, the mitochondrial apoptosis pathway is a key step in the intrinsic apoptotic pathway which turns on the caspase cascade. Hence, we further examined the expression level of caspase- 3 and caspase- 9 in the A375 cells. As shown in Fig. 6e, pro-caspase- 3 and pro-caspase9 decreased significantly in the A375 cells exposed to the $\alpha M / M P E G-P C L$ nanomicelles. Simultaneously, the cleaved-caspase- 3 and cleaved-caspase- 9 increased in a dose-dependent manner.

Finally, we detected the expression quantity alteration of caspase-8, a performer in exogenous apoptosis pathway. As shown in Fig. 6f, pro-caspase-8 level was downregulated and the expression quantity of cleaved-caspase8 was increased in A375 cells treated by the $\alpha \mathrm{M} / \mathrm{MPEG}$ PCL nanomicelles.

The proliferation and migration caused by the $\alpha M /$ MPEG-PCL nanomicelles in the human umbilical vein endothelial (HUVEC) cells were also studied to explore the preliminary anti-angiogenic mechanism. As shown in Figure S1, the $\alpha \mathrm{M} / \mathrm{MPEG}-\mathrm{PCL}$ nanomicelles inhibited the proliferation and suppressed the migration in a dose- and time-dependent manner.

Based on the above evaluations, we hypothesized that the $\alpha M / M P E G-P C L$ nanomicelles triggered apoptosis via both the mitochondrial-mediated intrinsic pathway and the exogenous apoptosis pathway. The $\alpha \mathrm{M} / \mathrm{MPEG}-\mathrm{PCL}$ nanomicelles also exhibited potential anti-angiogenic effect.

\section{In vivo anti-cancer activity of the aM nanomicelles}

Mice subcutaneous melanoma cancer model was employed to evaluate the effect of the $\alpha M / M P E G-P C L$ nanomicelles on anti-tumor activity. Tumor-bearing mice were assigned casually to four groups $(7 \mathrm{mice} /$ group), and treated with the normal saline (NS), the MPEG-PCL, the $\alpha M$, and the $\alpha M / M P E G-P C L$ nanomicelles, respectively, via intravenous injection. The body weight of mice and the volume of tumor were measured every 3 days. The tumor volume curves of each group were showed in Fig. 7a. The results indicated that the $\alpha \mathrm{M} / \mathrm{MPEG}-\mathrm{PCL}$ nanomicelles possessed better effect on tumor growth inhibition compared to the free $\alpha M$. Moreover, there was no obvious effect on the NS and MPEG-PCL groups. More specifically, the body weight 


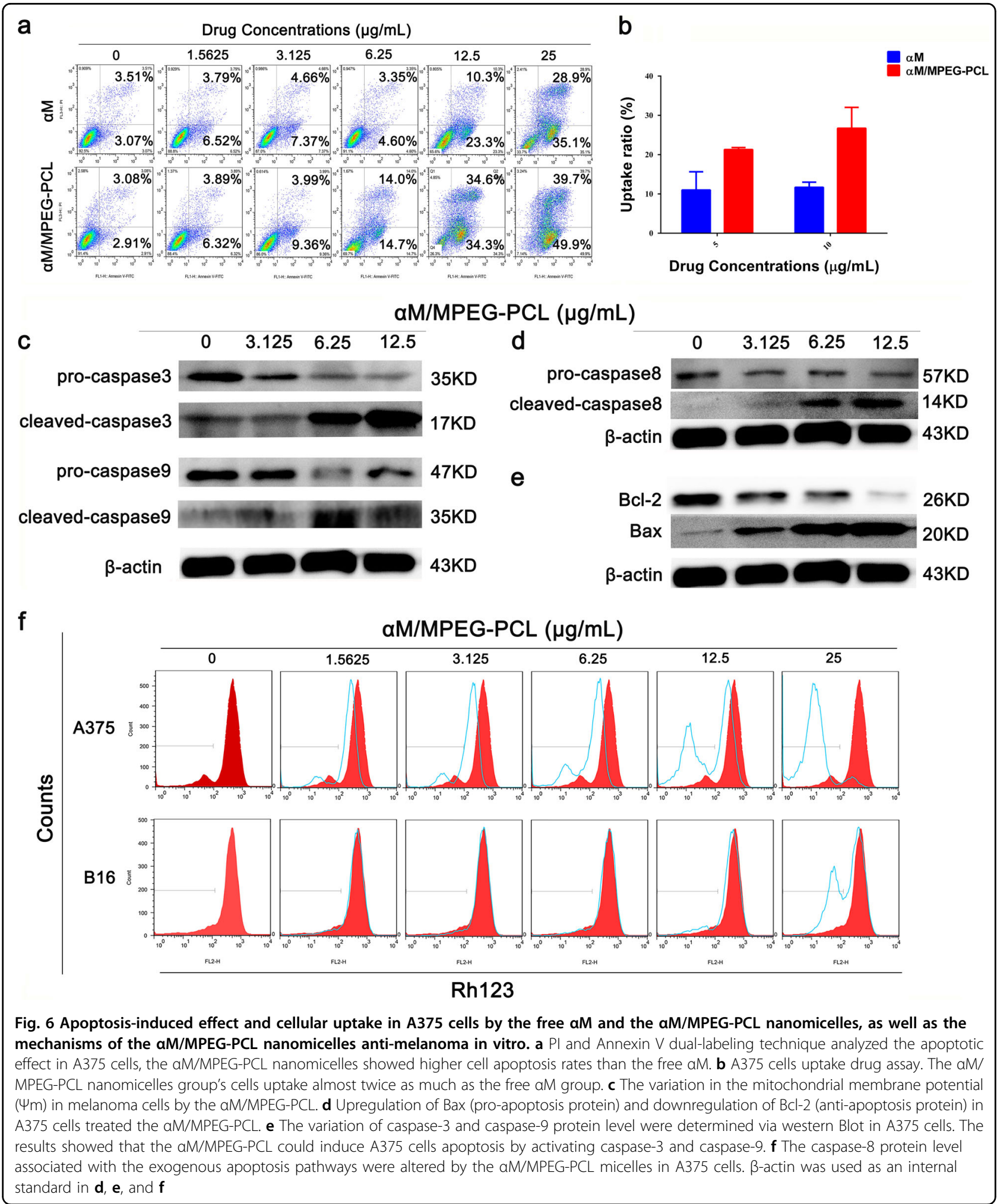

and tumor weight of the four groups were presented in Fig. 7b and c, respectively, as well as the tumor photos demonstrated in Fig. 7d. We evidently observed that the group treated with the $\alpha \mathrm{M} / \mathrm{MPEG}-\mathrm{PCL}$ nanomicelles held smaller tumor than those in the other groups. In conclusion, the strategy that encapsulated the $\alpha M$ into the MPEG-PCL nanomicelles comprehensively enhanced the anti-tumor activity of $\alpha \mathrm{M}$ in vivo. 


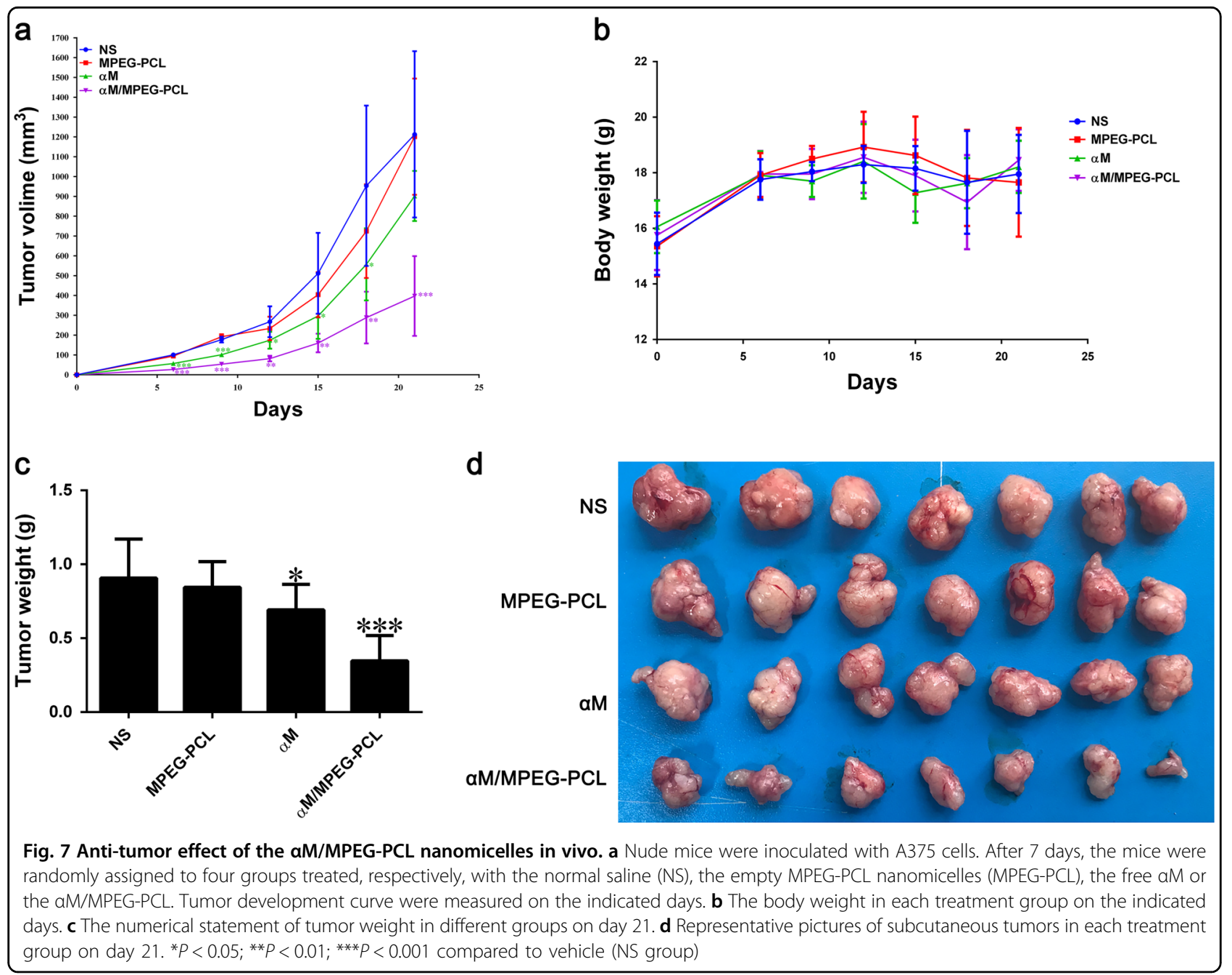

\section{Immunohistochemical analysis}

In order to detect the effect of the $\alpha M / M P E G-P C L$ nanomicelles anti-angiogenesis in frozen tumor sections, we performed an immunohistochemical analysis using antiCD31 monoclonal antibody. As demonstrated in Fig. 8a, CD31-positive endothelial cells in the group of the $\alpha \mathrm{M} /$ MPEG-PCL nanomicelles were remarkably less than that of the $\alpha M$, which was measured more CD31-positive endothelial cells than NS and MPEG-PCL nanomicelles groups.

Moreover, in order to confirm the effect of the $\alpha \mathrm{M} /$ MPEG-PCL nanomicelles on the melanoma cell proliferation, we performed proliferating cell nuclear antigen (PCNA) stained. In short, the tumor sections of each group were stained with PCNA and examined under a microscope. The iconic images were shown in Fig. 8b. The PCNA-positive tumor cells in the group of $\alpha \mathrm{M} / \mathrm{MPEG}$ PCL nanomicelles were markedly lower than the ones in other groups. The results suggested that the $\alpha M / M P E G-$ PCL nanomicelles significantly suppressed the proliferation of melanoma in vivo.
Simultaneously, we demonstrated that the $\alpha \mathrm{M} /$ MPEG-PCL nanomicelles-induced melanoma cells apoptosis in vitro. In order to further validate the mechanisms in vivo and explore whether the nanomicelles enhanced the activity of the $\alpha M$ against melanoma, the tumor sections were detected a terminaldeoxynucleoitidyl transferase mediated nick end labeling (TUNEL) assay. As shown in Fig. 8c, it was extremely obvious that the tumor samples treated with the $\alpha M$ and the $\alpha M / M P E G-P C L$ nanomicelles existed more significant cellular apoptosis than other groups. In addition, there were more apoptosis cells in the $\alpha \mathrm{M} /$ MPEG-PCL nanomicelles group than the $\alpha \mathrm{M}$ group. The apoptosis index in the $\alpha M / M P E G-P C L$ nanomicelles group was more remarkable than the one in the $\alpha M$ group, MPEG-PCL group and NS group, which implied the $\alpha M / M P E G-P C L$ nanomicelles-induced stronger apoptosis of melanoma in vivo compared with the $\alpha \mathrm{M}$. 


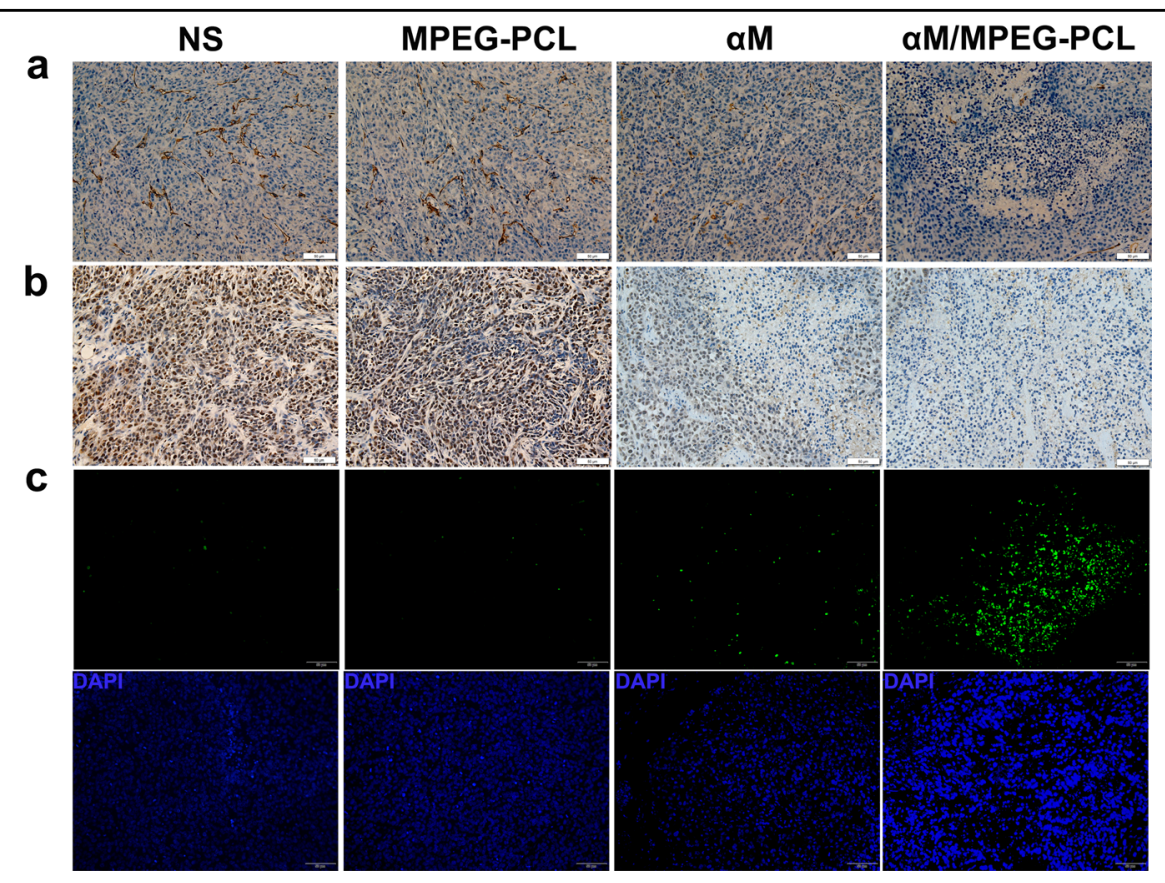

Fig. 8 Immunohistochemistry staining of tumor sections for the research of mechanisms of anti-melanoma in vivo. a CD31 immunofluorescence staining assay. Tumor tissues sections from four groups treated with the normal saline (NS), the empty MEPG-PCL nanomicelles (MPEG-PCL), the free $\mathrm{aM}(\mathrm{aM})$, and the aM/MPEG-PCL nanomicelles (aM/MPEG-PCL) were subjected to immunohistochemistry to analyze antiangiogenesis effect. $\mathbf{b}$ PCNA immunohistochemical staining analysis. Tumor sections from four groups (NS, MPEG-PCL, aM, aM/MPEG-PCL) were analyzed to PCNA immunohistochemical to detect tumor cell proliferation. c TUNEL assay. The apoptosis of A375 tumor sections was measured via TUNEL staining. The group treated with the aM/MPEG-PCL nanomicelles showed the most apoptotic cells, which demonstrated that the MPEG-PCL nanomicelles could improve the anti-tumor activity of the aM

\section{Safety profile of the aM/MPEG-PCL nanomicelles}

To further investigate the safety profile of the $\alpha \mathrm{M} /$ MPEG-PCL nanomicelles, we performed several assays, including blood routine examination, blood chemistry test, and Hematoxylin-Eosin (HE) staining analysis. Obviously, no pathological change was found after the $\alpha M / M P E G-P C L$ nanomicelles treatment in Figure S2 and S3. Moreover, in the Figure S4, no evidentially pathological morphologic change was observed after the $\alpha \mathrm{M} /$ MPEG-PCL nanomicelles therapy occurred in the lung, liver, heart, kidney, and spleen via microscopy study compared with the control group.

\section{Discussion}

As a traditional and popular natural medicine, the $\alpha M$ has possessed promising application to treat various diseases $^{24,25}$. Previous studies have shown that the $\alpha M$ offered good therapeutic effect on a series of cancers ${ }^{22-24}$. Nevertheless, the clinical application is gravely limited because of hydrophobicity. In this study, the MPEG-PCL nanomicelles were successfully applied in resolving the poor aqueous solubility of the $\alpha M$ and enhancing the activity of anti-melanoma.

Currently, nanometer material has been reported to be applied to heighten the solubility and strengthen the biological availability of hydrophobic drug. As a promising biodegradable nanometer material, the MPEG-PCL nanomicelles has been widely used to deliver drugs for tumor therapy owing to its outstanding drug entrapment efficiency (EE), high stability and hypotoxicity ${ }^{12}$. In this study, we developed the $\alpha M / M P E G-P C L$ nanomicelles via a one-step solvent evaporation way. The $\alpha M$ was wrapped in the MPEG-PCL nanomicelles to improve its water solubility, which obtained a better anti-melanoma activity. Furthermore, we compared with the free $\alpha M$ and the $\alpha M$ / MPEG-PCL nanomicelles on the effect of anti-melanoma as well as probed the mechanisms of action of the $\alpha \mathrm{M} / \mathrm{MPEG}-\mathrm{PCL}$ nanomicelles.

In our survey, the $\alpha M / M P E G-P C L$ nanomicelles were provided with stable drug loading and highencapsulation efficiency. Meanwhile, the characterization of the $\alpha \mathrm{M} / \mathrm{MPEG}-\mathrm{PCL}$ nanomicelles illustrated small particle size and nearly neutral surface charge (zeta potential $=-2.1 \mathrm{mv}$ ), as well as monodisperse suspension in aqueous phase owing to the core-shell structure of polymeric nanomicelles. In addition, the nanostructure of the nanomicelles was monitored by TEM, which implied that the prepared $\alpha \mathrm{M} / \mathrm{MPEG}-\mathrm{PCL}$ nanomicelles were solvable and stable in aqueous solution. In previous studies, nanomicelles, permeability, and retention (EPR) 
effect were enhanced if the drug-loaded nanomicelles possessed suitable size $(10-100 \mathrm{~nm})$, and consequently, the accumulation in cancer tissue increased anticarcinoma effects ${ }^{26,27}$. Besides, the release behavior of the presented highly sustained drug was realized in the $\alpha M$ nanomicelles owing to the tiny size and the stable hydrophilic interface of the MPEG-PCL nanomicelles. Furthermore, the results of drug-release experiments in vitro illustrated the MPEG-PCL nanomicelles prolonged the duration of the $\alpha \mathrm{M}$ release. Meanwhile, in the study of pharmacokinetics analysis in vivo, the plasma concentrations of the $\alpha \mathrm{M} / \mathrm{MPEG}-\mathrm{PCL}$ nanomicelles group markedly reduced than the $\alpha \mathrm{M}$ groups. The $\alpha \mathrm{M} /$ MPEG-PCL nanomicelles were obviously observed that it extended the $\alpha \mathrm{M}$ treating duration to improve the effectiveness by sustaining release. The results indicated the $\alpha \mathrm{M} / \mathrm{MPEG}-\mathrm{PCL}$ nanomicelles possessed excellent antineoplastic potentials.

Meanwhile, a series of experiments were performed to assess the anti-melanoma effect of the $\alpha \mathrm{M} / \mathrm{MPEG}-\mathrm{PCL}$ nanomicelles compared with the free $\alpha \mathrm{M}$, including both in vitro and in vivo assays. We hypothesized some antimelanoma mechanisms for the $\alpha M / M P E G-P C L$ nanomicelles. First, in the cytotoxicity tests, we found the $\alpha M /$ MPEG-PCL nanomicelles showed a better inhibitory effect on the growth and proliferation of the melanoma cells (A375 and B16 cells) than the free $\alpha \mathrm{M}$ via MTT assay and colony formation experiments. Meanwhile, the $\alpha \mathrm{M} /$ MPEG-PCL nanomicelles showed a lower toxicity in the non-tumor cell lines, including HEK293T, LO2, and Vero cells, compared with the melanoma cells. Moreover, the $\alpha M / M P E G-P C L$ nanomicelles induced the apoptosis in the melanoma cells via FCM assay. The above results demonstrated the $\alpha M / M P E G-P C L$ nanomicelles had more apoptosis ratio than the free $\alpha \mathrm{M}$. In addition, we found the $\alpha M$ with the encapsulation of the MPEG-PCL nanomicelles was more easily absorbed by melanoma cells, which contributed to achieving a higher concentration in cellular. Hence, we hypothesized that the enhancement effect of anti-melanoma activity was derived from the high uptake ratio of the $\alpha \mathrm{M} / \mathrm{MPEG}-\mathrm{PCL}$ nanomicelles in vitro.

These results suggested the $\alpha M / M P E G-P C L$ nanomicelles suppressed melanoma cells growth retardation associated with apoptosis-inducing process. It is wellknown that apoptosis plays a vital role in the development and maintenance of tissue homeostasis as well as is indispensable function for the initiation and progression of cancer ${ }^{28-30}$, including the mitochondrial (intrinsic) and death receptor pathways (extrinsic) ${ }^{31}$. In the process of exploring the potential mechanisms of the $\alpha M / M P E G-$ PCL nanomicelles-induced apoptosis in melanoma, we found the variation of mitochondrial membrane potential (MMP) with Rh123 stained and the expression of a series of apoptosis-associated proteins with WB. Experimental results validated the $\alpha M / M P E G-P C L$ nanomicelles significantly declined the MMP, which is one of the major characteristics of the mitochondrial-induced apoptosis pathway $^{32}$. Moreover, the $\alpha$ M/MPEG-PCL nanomicelles enhanced the expression of Bax (pro-apoptotic protein) while decreased the expression of $\mathrm{Bcl}-2$ (anti-apoptotic protein $)^{33}$. Furthermore, we found the reduction in the expression of pro-caspase- 9 and pro-caspase- 8 as well as the augment with the expression of cleaved-caspase- 9 and cleaved-caspase-8 in WB experiments. More importantly, after incubation with the $\alpha M / M P E G-P C L$ nanomicelles, the downregulated expression of pro-caspase-3 and the upregulated expression of cleaved-caspase- 3 were observed in A375 cells. It is well-known that caspase-3, an executioner caspase ${ }^{34}$, is the end of the stage of apoptosis commencer shared by the both pathways ${ }^{35,36}$. Consequently, our research indicated that the $\alpha \mathrm{M} / \mathrm{MPEG}-\mathrm{PCL}$ nanomicelles induced apoptosis process in the melanoma cells via mitochondrial-mediated endogenous pathway and extrinsic apoptosis pathway. In the nude mouse model injected A375 cells, our results demonstrated that the weights and volumes of tumors were more significantly suppressed in the $\alpha M / M P E G-P C L$ nanomicelles group compared with any another group. Simultaneously, no obvious changes on the body weight of nude mice were found, which indicated the $\alpha \mathrm{M} / \mathrm{MPEG}-\mathrm{PCL}$ nanomicelles had stronger anti-melanoma effect and exhibited low cytotoxicity.

Furthermore, the possible mechanisms of the antimelanoma activity of the $\alpha \mathrm{M} / \mathrm{MPEG}-\mathrm{PCL}$ nanomicelles in vivo were proposed. It was reported that the expression of PCNA was closely related to the proliferation ${ }^{37}$. As shown in Fig. 8b, the PCNA-positive cells in the $\alpha \mathrm{M} /$ MPEG-PCL nanomicelles group were much fewer than the ones in the other groups, suggesting the $\alpha M / M P E G-$ PCL nanomicelles blocked melanoma associated with cell proliferation suppression and possessed better effect compared to the free $\alpha \mathrm{M}$. Furthermore, angiogenesis is crucial in tumor growth and anti-angiogenic, which is considered as a promising therapeutic strategy for tumor treatment $^{38,39}$. Hence, we implemented the immunohistochemical analysis of CD31. The results showed that through the $\alpha M / M P E G-P C L$ nanomicelles treatment presented fewer angiogenesis effects than the free $\alpha \mathrm{M}$, NS, and MPEG-PCL groups in tumor tissue, which indeed proved the $\alpha \mathrm{M} / \mathrm{MPEG}-\mathrm{PCL}$ nanomicelles exerted the anti-melanoma effect involving in the distinct impairment of neovascularization. Indeed, the $\alpha \mathrm{M} /$ MPEG-PCL nanomicelles could possess the inhibition on cell viability and cell migration of the HUVEC cells in vitro. Additionally, the apoptosis tumor cells were detected with TUNEL staining in vivo and showed in Fig. $8 c$. On the basis of the results, the $\alpha$ M/MPEG-PCL 
nanomicelles induced a higher apoptosis ratio than the free $\alpha \mathrm{M}$.

In conclusion, the $\alpha M / M P E G-P C L$ nanomicelles were developed by a thin-film hydration method and applied to the therapy of melanoma in vitro and in vivo. The $\alpha \mathrm{M} /$ MPEG-PCL nanomicelles with a high EE and controlled release behavior in vitro possessed more excellent cellular uptake, cytotoxicity, proliferation inhibition, and apoptosisinducing in the melanoma cells than the free $\alpha \mathrm{M}$. Moreover, a slow excretion behavior from blood vessels was also observed in the $\alpha \mathrm{M} / \mathrm{MPEG}-\mathrm{PCL}$ nanomicelles group. Besides, in A375 cell-bearing tumor model, the $\alpha$ M/MPEGPCL nanomicelles more dramatically inhibited tumor growth and angiogenesis than other groups (including the free $\alpha M)$. In the exploration of mechanisms, we found the $\alpha \mathrm{M} / \mathrm{MPEG}-\mathrm{PCL}$ nanomicelles repressed the melanoma cells via the mitochondrial-mediated intrinsic pathway and the exogenous apoptosis pathway. In short, the $\alpha \mathrm{M} / \mathrm{MPEG}$ PCL nanomicelles presented in this work demonstrated an improved anti-melanoma activity both in vitro and in vivo, which suggested this novel formulation had potential applications in the melanoma therapy.

\section{Methods}

\section{Preparation of aM/MPEG-PCL nanomicelles}

For preparation of the $\alpha M / M P E G-P C L$ nanomicelles, $10 \mathrm{mg} \alpha \mathrm{M}$ and $90 \mathrm{mg}$ MPEG-PCL copolymer were resuspended in $10 \mathrm{~mL}$ methanol with suitably stirring. Subsequently, the methanol was removed by a rotary evaporator at $50^{\circ} \mathrm{C}$. During the process, an amorphous substance was obtained including the $\alpha \mathrm{M}$ and the MPEGPCL. Meanwhile, the $\alpha M$ was dissolved into MPEG-PCL copolymer to get an amorphous substance. Then, nanomicellesthe co-evaporation was dissolved in Milli-Q water at $55^{\circ} \mathrm{C}$ to achieve the $\alpha \mathrm{M} / \mathrm{MPEG}-\mathrm{PCL}$ nanomicelles by self-assembly method. Finally, the produce of the $\alpha \mathrm{M} /$ MPEG-PCL nanomicelles solution with a concentration of $5 \mathrm{mg} / \mathrm{mL}$ was filtered by $0.22 \mu \mathrm{m}$ syringe filter (Jinteng, Tianjin, China), lyophilized and stored at $4{ }^{\circ} \mathrm{C}$ for future use.

\section{Molecular modeling study of the interaction between the $\mathrm{aM}$ and the copolymer MPEG-PCL}

The interaction between the $\alpha \mathrm{M}$ and the copolymer MPEG-PCL was simulated. First, the 3D structures of the $\alpha \mathrm{M}$ and the copolymer were fabricated by the same ways as described in previous studies ${ }^{12}$. After that, the $\alpha \mathrm{M}$ was docked randomly to the simulated copolymer MPEG-PCL by merging the $\alpha \mathrm{M}$ to the copolymer in the workspace of HyperChem. After that, the $\alpha \mathrm{M} / \mathrm{MPEG}-\mathrm{PCL}$ complex experienced 2 stage Langevin Dynamics simulation.

At the first stage of simulation, the interaction between the $\alpha \mathrm{M}$ and the copolymer MPEG-PCL was simulated, and the solvation effect was considered implicitly by setting the scale factor for the dielectric permittivity to 80 . At the second stage, the complex was further simulated in aqueous solution and in tumor tissue, respectively. The interaction between the $\alpha \mathrm{M}$ and the MPEG-PCL near the tumor tissue was simulated by setting the scale factor to 26 . At each stage, the running time was set to $100 \mathrm{ps}$ and the temperature, friction coefficient, and random seed were set to $300 \mathrm{~K}, 0.075 \mathrm{ps}^{-1}$, and 0 , respectively. CHARMM27 was chosen as the force field.

\section{In vitro release}

Release behaviors of the free $\alpha M$ and the $\alpha$ M/MPEGPCL nanomicelles were evaluated by dialysis with a molecular cutoff $3.5 \mathrm{KDa}$. Briefly, $1 \mathrm{~mL}$ drug solution (the free $\alpha M$ or the $\alpha M / M P E G-P C L$ nanomicelles) was transferred in dialysis bags suspended in in $40 \mathrm{~mL}$ PBS $(\mathrm{pH}=7.4)$ containing Tween $80(0.5 \%, \mathrm{w} / \mathrm{w})$ at $37^{\circ} \mathrm{C}$. (Sigma-Aldrich Co.). The whole device was placed in a shaking water with gentle shaking $(100 \mathrm{rpm})$. After that, at predetermined time intervals, $200 \mu \mathrm{L}$ release medium samples were collected and supplemented with an equal volume of fresh medium. The amount of released $\alpha \mathrm{M}$ was quantitated by HPLC. The experiment was repeated three times and the results were expressed as mean \pm SD.

\section{Pharmacokinetic}

The Sprague Dawley (SD) rats were randomly divided into two groups: the free $\alpha M$ and the $\alpha M / M P E G-P C L$ nanomicelles. Both groups were, respectively, dissolved into the mixed solution containing Tween 80 and dehydrated alcohol $(1: 1, \mathrm{v} / \mathrm{v})$ and the normal saline (NS). After starved overnight, the rats received a dose of $50 \mathrm{mg} / \mathrm{kg}$ drugs administered intravenously. Then, the blood was collected by a capillary from eye sockets at various predetermined intervals ( $5 \mathrm{~min}, 15 \mathrm{~min}, 30 \mathrm{~min}, 45 \mathrm{~min}, 1 \mathrm{~h}$, $2 \mathrm{~h}, 4 \mathrm{~h}, 6 \mathrm{~h}$, and $8 \mathrm{~h}$; three mice each time point). The blood samples were extracted by methanol, evaporated, and stored at $-20^{\circ} \mathrm{C}$ until LC-MS analysis.

\section{Cell proliferation}

A375, B16, and HUVEC cells were seeded in 96-well plates at $2.5-5 \times 10^{3}$ cells/well. Gradient concentrations of the $\alpha \mathrm{M}$ and the $\alpha \mathrm{M} / \mathrm{MPEG}-\mathrm{PCL}$ nanomicelles were added to the plates for specific time. Twenty microliters of MTT working solution $(5 \mathrm{mg} / \mathrm{mL})$ was added to each well. After $4 \mathrm{~h}$ incubation at $37^{\circ} \mathrm{C}$, the supernatant was removed and $150 \mu \mathrm{L}$ dimethyl sulfoxide (DMSO) per well was appended. The plates laid up a shaker for about $10 \mathrm{~min}$. The optical density was measured via Spectra MAX M5 microplate spectrophotometer at $570 \mathrm{~nm}$.

\section{Colony formation assay}

A total of $300-500$ cells (A375 or B16 cells) were plated in a 6-well plate. After 3 days incubation, the cells were 
treated with diverse concentrations of the $\alpha M$ and the $\alpha$ M/MPEG-PCL nanomicelles for 15 days. The culture medium was replaced with a fresh medium containing same concentrations of drug every 3 days. After colonies were formed, the cells were stained with crystal violet and fixed by methanol.

\section{Cells apoptotic assay by FCM}

The A375 and B16 cells were treated with the $\alpha \mathrm{M}$ and the $\alpha M / M P E G-P C L$ for $24 \mathrm{~h}$ and collected in the flow tubes. After adding the PI and Annexin V into the flow tubes in accordance with the usage instructions, we detected the level of apoptosis via FCM (BD Bioscience). Finally, the FlowJo software was used to analyze the data that represented the average of independent experiments.

\section{Examination of mitochondrial membrane potential $(\Psi \mathrm{m})$}

A375 and B16 cells were treated with indicated doses of the $\alpha M / M P E G-P C L$ for $24 \mathrm{~h}$ incubated with $5 \mu \mathrm{g} / \mathrm{mL}$ Rhodamine $123(\mathrm{Rh} 123)$ at $37^{\circ} \mathrm{C}$ in the dark for $30 \mathrm{~min}$. The stained cells were collected and washed twice with cold PBS, and then the fluorescence was subsequently detected by FCM.

\section{Cellular uptake}

A375 cells were grown in complete medium to reach $80 \%$ confluence. After removing the original medium, the cells were treated with the $\alpha \mathrm{M}(5 \mu \mathrm{g} / \mathrm{mL}, 10 \mu \mathrm{g} / \mathrm{mL})$ and the $\alpha$ M/MPEG-PCL $(5 \mu \mathrm{g} / \mathrm{mL}, 10 \mu \mathrm{g} / \mathrm{mL})$ for $3 \mathrm{~h}$. The cells were collected and washed for three times by PBS. Then the cells were re-suspended in methanol and sonicated to collect the drug from cellular. The supernatants were collected after twice centrifugation $(13,000 \mathrm{rpm}$, $10 \mathrm{~min})$. Finally, the drug content was determined by HPLC. The test was performed in triplicate.

\section{Western blot analysis}

A375 cells treated with various concentrations of the $\alpha M / M P E G-P C L$ were lysed in RIPA buffer (Beyotime, Beijing, China). The total proteins were quantified by Nanodrop $^{\text {Tw }}$ 2000/2000c spectrophotometers (Thermo Fisher Scientific, Waltham, Massachusetts, USA) and equalized before loading. Equal amounts of protein from each sample were fractionated by sodium dodecyl sulphate polyacrylamide gel (SDS-PAGE) electrophoresis and transferred onto polyvinylidene difluoride (PVDF) membrane accordingly. Then, the membranes were blocked with $5 \%$ bovine serum albumin (BSA) for $2 \mathrm{~h}$ at $37^{\circ} \mathrm{C}$ and incubated with homologous primary antibody working solution overnight at $4{ }^{\circ} \mathrm{C}$. After washed three times ( 15 min per time) by tris-buffered saline with Tween 20 (TBST), the membranes were incubated with corresponding secondary antibody with a working concentration based on its specification at $37^{\circ} \mathrm{C}$ for $1 \mathrm{~h}$. Finally, the spots of proteins were measured via an enhanced chemiluminescence system (Amersham Biosciences, Buckinghamshire, UK). $\beta$-actin was regarded as a reference.

\section{Evaluation of the anti-melanoma effect of the aM/MPEG- PCL nanomicelles in vivo}

A375 cells $\left(1 \times 10^{7}\right)$ in $0.1 \mathrm{~mL}$ serum-free medium were injected subcutaneously into the female BALB/c athymic nude mice. Mice were randomly assigned into four groups (7 mice/group) when the tumor volume reached $\sim 100 \mathrm{~mm}^{3}$. After that, the four groups were treated with the NS, the MPEG-PCL, the free $\alpha M(50 \mathrm{mg} / \mathrm{kg})$ or the $\alpha M / M P E G-P C L \quad(50 \mathrm{mg} / \mathrm{kg})$, respectively. The tumor volumes were measured every 3 days with a vernier caliper and tumor volumes were calculated according to the equation: $V=L \times W^{2} \times 0.52$. Moreover, the body weight of each mouse was measured every 3 days and treatment conditions were observed daily. At the termination of the experiment, mice were killed by cervical dislocation. In addition, tumor samples were collected and weighed.

\section{TUNEL assay}

The tumors were fixed in PBS containing $4 \%$ paraformaldehyde for at least $24 \mathrm{~h}$, following by exposure in $70 \%$ ethanol overnight and embedded in paraffin. The sections (3-5 $\mu \mathrm{m}$ thick) were cut and mounted. A commercially procurable terminal-deoxynucleoitidyl transferase mediated nick end labeling (TUNEL, Promega, Madison, WI, USA) was applied to detect apoptotic cells within A375 tumor cells. The TUNEL assay was carried out according to the manufacturer's protocol and the samples were examined with a fluorescence microscope $(\times 400)$.

\section{Immunohistochemical (IHC) determination of PCNA and CD31}

The expression of PCNA and CD31 were estimated by immunochemistry with PCNA and CD31 antibodies. Briefly, the frozen tumor tissues were fixed in acetone and washed twice with PBS. Following, the handled samples were stained with rabbit anti-mouse CD31 monoclonal antibody (1:50; Abcam, Cambridge, UK) and rabbit antirat PCNA polyclonal antibody (1:50; BD Pharmingen ${ }^{\mathrm{TM}}$; BD Biosciences, San Jose, CA, USA). After washing twice with PBS, samples were stained with secondary antibody combining with FITC or Rhodamine. Finally, we surveyed the cells possessing-positive property under microscope $(\times 400)$ and counted automatically the number of capillaries in 5 randomly selected fields.

\section{Statistical analysis}

Data of three-independent experiments were expressed as the mean \pm standard deviation (SD). Statistical analysis was performed with Graph Pad and Excel. Student's test 
was employed for comparing treatment and control groups. $P$-values were marked as follows: ${ }^{*} P<0.05$; ${ }^{* *} P<$ $0.01 ; * * * 0.001$.

\section{Acknowledgements}

This work was supported by the National Natural Science Foundation of China (NSFC81502165), Ministry of Science and Technology of the People's Republic of China (2018ZX09201018-005) and Guangdong Innovative Research Team Program (No. 2011Y073).

\section{Author contributions}

S.Y. and X.G. conducted all experiments and prepared the manuscript with the help of M.X. and Y.X.; Y.H., and Y.H. performed immunohistochemical determination of PCNA and CD31; B.X. and Z.C. helped edit the manuscript and analyze the data.

\section{Conflict of interest}

The authors declare that they have no conflict of interest.

\section{Publisher's note}

Springer Nature remains neutral with regard to jurisdictional claims in published maps and institutional affiliations.

Supplementary Information accompanies this paper at (https://doi.org/ 10.1038/s41419-019-1323-9).

Received: 27 June 2018 Revised: 20 December 2018 Accepted: 7 January 2019

Published online: 15 February 2019

\section{References}

1. Guinée, J. B., Heijungs, R., Vijver, M. G. \& Wjgm, P. Setting the stage for debating the roles of risk assessment and life-cycle assessment of engineered nanomaterials. Nat. Nanotechnol. 12, 727-733 (2017).

2. Gao, Y. et al. China and the United States-Global partners, competitors and collaborators in nanotechnology development. Nanomed. Nanotechnol. 12 13-19 (2016).

3. D'Mello, S. R. et al. The evolving landscape of drug products containing nanomaterials in the United States. Nat. Nanotechnol. 12, 523-529 (2017).

4. Jang, C., Lee, J. H., Sahu, A. \& Tae, G. The synergistic effect of folate and RGD dual ligand of nanographene oxide on tumor targeting and photothermal therapy in vivo. Nanoscale 7, 18584-18594 (2015).

5. Jin, Y. et al. Biodegradable, multifunctional DNAzyme nanoflowers for enhanced cancer therapy. Npg Asia Mater. 9, e365 (2017).

6. Chen, Y., Wu, Y., Sun, B., Liu, S., \& Liu, H. Two-dimensional nanomaterials for cancer nanotheranostics. Small 13, 1603446 (2017).

7. Chen, H., Zhang, W., Zhu, G., Xie, J., \& Chen, X. Rethinking cancer nanotheranostics. Nat. Rev. Mater. 2, 17024 (2017).

8. Malam, Y., Loizidou, M. \& Seifalian, A. M. Liposomes and nanoparticles: nanosized vehicles for drug delivery in cancer. Trends Pharmacol. Sci. 30 , 592-599 (2009).

9. Chen, B. L. et al. Current multistage drug delivery systems based on the tumor microenvironment. Theranostics 7, 538-558 (2017).

10. Hu, Q., Sun, W., Chao, W. \& Zhen, G. Recent advances of cocktail chemotherapy by combination drug delivery systems. Adv. Drug Deliv. Rev. 98, 19-34 (2016).

11. Weng, Y. H. et al. Nanomicelle-assisted targeted ocular delivery with enhanced antiinflammatory efficacy in vivo. Adv. Sci. 5, 1700455 (2018).

12. Gao, X. et al. Improving the anti-ovarian cancer activity of docetaxel with biodegradable self-assemblymicelles through various evaluations. Biomaterials 53, 646-658 (2015).
13. Parrott, M. C. \& Desimone, J. M. Drug delivery: relieving PEGylation. Nat. Chem. 4, 13-14 (2012).

14. Shay, J. W. New insights into melanoma development. Science $\mathbf{3 5 7}$ 1358-1359 (2017)

15. Dorard, C. et al. RAF proteins exert both specific and compensatory functions during tumour progression of NRAS-driven melanoma. Nat. Commun. 8, 15262 (2017).

16. Long, G. V. et al. Adjuvant dabrafenib plus trametinib in stage III BRAF-mutated melanoma. New Engl. J. Med. 377, 1813-1823 (2017).

17. Siegel, R. L., Miller, K. D. \& Jemal, A. Cancer statistics, 2017. Cancer J. Clin. 67, 7-30 (2017).

18. Perego, M. et al. A slow-cycling subpopulation of melanoma cells with highly invasive properties. Oncogene 37, 302-312 (2018).

19. Li, P., Tian, W., \& Ma, X. Alpha-mangostin inhibits intracellular fatty acid synthase and induces apoptosis in breast cancer cells. Mol. Cancer 13, 138 (2014)

20. Johnson, J. J. et al. alpha-Mangostin, a xanthone from mangosteen fruit, promotes cell cycle arrest in prostate cancer and decreases xenograft tumor growth. Carcinogenesis 33, 413-419 138 (2012).

21. Limon, J. J., Skalski, J. H. \& Underhill, D. M. Commensal fungi in health and disease. Cell Host Microbe 22, 156-165 (2017).

22. Silva, L. N., Zimmer, K. R., Macedo, A. J. \& Trentin, D. S. Plant natural products targeting bacterial virulence factors. Chem. Rev. 116, 9162-9236 (2016).

23. Shibata, M. A. et al. alpha-Mangostin extracted from the pericarp of the mangosteen (Garcinia mangostana Linn) reduces tumor growth and lymph node metastasis in an immunocompetent xenograft model of metastatic mammary cancer carrying a p53 mutation. BMC Med. 9, 69 (2011).

24. Smallwood, H. S. et al. Targeting metabolic reprogramming by influenza infection for therapeutic intervention. Cell Rep. 19, 1640-1653 (2017).

25. Lin, S. M. et al. Semisynthesis and biological evaluation of xanthone amphiphilics as selective, highly potent antifungal agents to combat fungal resistance. J. Med. Chem. 60, 10135-10150 (2017).

26. Björnmalm, M., Thurecht, K. J., Michael, M., Scott, A. M. \& Caruso, F. Bridging bio-nano science and cancer nanomedicine. ACS nano. 11, 9594-9613 (2017)

27. Shen, L. Y. et al. pH-responsive aerobic nanoparticles for effective photodynamic therapy. Theranostics 7, 4537-4550 (2017).

28. Kumar, S., Calianese, D. \& Birge, R. B. Efferocytosis of dying cells differentially modulate immunological outcomes in tumor microenvironment. Immunol. Rev. 280, 149-164 (2017).

29. Childs, B. G., Baker, D. J., Kirkland, J. L., Campisi, J. \& Deursen, J. M. Senescence and apoptosis: dueling or complementary cell fates? EMBO Rep. 15, 1139-1153 (2014).

30. Giampazolias, E. et al. Mitochondrial permeabilization engages NF-kBdependent anti-tumour activity under caspase deficiency. Nat. Cell Biol. 19, 1116-1129 (2017).

31. Zhang, Y., Chen, X., Gueydan, C. \& Han, J. Plasma membrane changes during programmed cell deaths. Cell Res. 28, 9-21 (2018).

32. Chipuk, J. E. et al. Sphingolipid metabolism cooperates with BAK and BAX to promote the mitochondrial pathway of apoptosis. Cell 148, 988-1000 (2012).

33. Ashkenazi, A., Fairbrother, W. J., Leverson, J. D. \& Souers, A. J. From basic apoptosis discoveries to advanced selective BCL-2 family inhibitors. Nat. Rev. Drug Discov. 16, 273-284 (2017).

34. Man, N. et al. Caspase-3 controls AML1-ETO-driven leukemogenesis via autophagy modulation in a ULK1-dependent manner. Blood 129, 2782-2792 (2017).

35. Nakajima, Y. I. \& Kuranaga, E. Caspase-dependent non-apoptotic processes in development. Cell Death Differ. 24, 1422-1430 (2017).

36. Márquezjurado, S. et al. Mitochondrial levels determine variability in cell death by modulating apoptotic gene expression. Nat. Commun. 9, 389 (2018).

37. Dilley, R. L. et al. Break-induced telomere synthesis underlies alternative telomere maintenance. Nature 539, 54-58 (2016).

38. Hugo, W. et al. Genomic and transcriptomic features of response to anti-PD-1 therapy in metastatic melanoma. Cell 168, 542 (2017).

39. Boareto, M., Jolly, M. K., Ben-Jacob, E. \& Onuchic, J. N. Jagged mediates differences in normal and tumor angiogenesis by affecting tip-stalk fate decision. Proc. Natl Acad. Sci. USA 112, E3836-E3844 (2015). 